\title{
Neutron Diffraction as a Probe of Microstructure: Surveying the Forest Before Examining the Trees
}

\author{
John H. Root* \\ *National Research Council of Canada, Chalk River Laboratories, Chalk River, Canada K0J 1J0
}

With the advancement of microscopy technologies in recent years, and the reasonable availability of instruments, researchers can acquire an impressive amount of information at the micro and nano scales. Neutron diffraction is not suitable for imaging small details. However, it is relatively straightforward with neutron diffraction to obtain the volume fractions of phases [1], crystallite orientation distribution functions [2] and the evolution of microstructures under load [3,4] or at elevated temperatures, to complement the understanding of phenomena deduced from microscopy techniques. For specimens that require complex environments to exhibit their relevant responses (e.g. some combination of pressure, temperature, hydration or chemical stress), neutron diffraction may be the only practical method to obtain any microstructure information [5]. This paper will review two examples where neutron diffraction measurements suggested novel ideas about microstructure evolution, and pointed the way for subsequent investigation by microscopy methods.

In Ref. 6, a metal-matrix composite of titanium carbide (TiC) particles in a titanium matrix was made by sintering mixed-powder compacted specimens in vacuum at temperatures between $1000^{\circ} \mathrm{C}$ and $1500^{\circ} \mathrm{C}$. By reaction with the metal matrix, $\mathrm{TiC}$ was converted to a carbon-depleted $\mathrm{TiC}_{\mathrm{x}}$, with a lattice parameter sufficiently reduced that its diffraction peaks could be resolved from those of $\mathrm{TiC}$ (Fig. 1a). The integrated intensities of these peaks were plotted versus time (Fig. 1b) to monitor the reaction between the carbide particles and the matrix during sintering. The observation of two distinct diffraction peaks during sintering (suggesting a single value of $\mathrm{x}$ ), rather than the gradual broadening of a single peak (continuous range of stoichiometry $1.0>\mathrm{x}>0.5$ ), led the authors to surmise that the microstructure of the carbide particles might exhibit a distinctive outer shell, depleted in carbon content. Low-voltage, high-resolution field emission gun scanning electron microscopy (FEGSEM) provided sufficient contrast to confirm the sharp boundary between the inner and outer regions of carbide particles.

In Ref. 7, neutron diffraction was applied to study the kinetics of a phase transformation in zirconium hydrides precipitated by cooling a zirconium alloy specimen (containing about $200 \mathrm{mg}$ deuterium per $\mathrm{kg}$ of zirconium) from $450^{\circ} \mathrm{C}$ to a holding temperature of $17^{\circ} \mathrm{C}$. Over 60 hours, the (111) diffraction peak of $\delta$-phase hydride, $\mathrm{ZrH}_{1.6}$, decreased while the (111) peak from the $\gamma$-phase hydride, $\mathrm{ZrH}$, increased as shown in Fig. 2. The authors noted that a transformation from the $\delta$ to the $\gamma$ phase would require diffusion of hydrogen out of the prior $\delta$-phase precipitates. They proposed that hydride precipitates might exhibit a core of untransformed $\delta$-phase and that $\gamma$-phase hydrides would be found at the interface between the hydride precipitate and the zirconium matrix. The $\gamma-$ and $\delta$-hydride phases can be distinguished by electron energy loss spectroscopy (EELS) in a transmission electron microscope [8]. A TEM micrograph, Fig. 3 [9], was obtained from a specimen of material from the neutron diffraction study, and the EELS analysis identified $\gamma$-phase hydrides that appeared to grow like dendrites out of the boundary of a prior $\delta$-phase precipitate. 


\section{References}

[1] H. Abuluwefa et al., Metall. and Mater. Trans. 27B (1996) 993.

[2] H.J. Bunge, Textures and Microstructures 10 (1989) 265.

[3] M.A. Gharghouri et al., Phil. Mag. A79 (1999) 1671.

[4] P. Dawson et al., Mater. Sci. Engng. A 313 (2001) 123.

[5] I.P. Swainson and E.M. Shulson, Cement and Concrete Research 31 (2001), 1821.

[6] P. Wanjara et al., Acta mater. 48 (2000) 1443.

[7] M. Small et al., J. Nucl. Mater. 256 (1998), 102.

[8] O.T. Woo and G.J.C. Carpenter, Microsc. Microanal. Microstruct. 3 (1992) 35.

[9] The TEM micrograph in Fig. 3 was obtained by O.T. Woo, Atomic Energy of Canada Limited, Chalk River Laboratories, Canada.

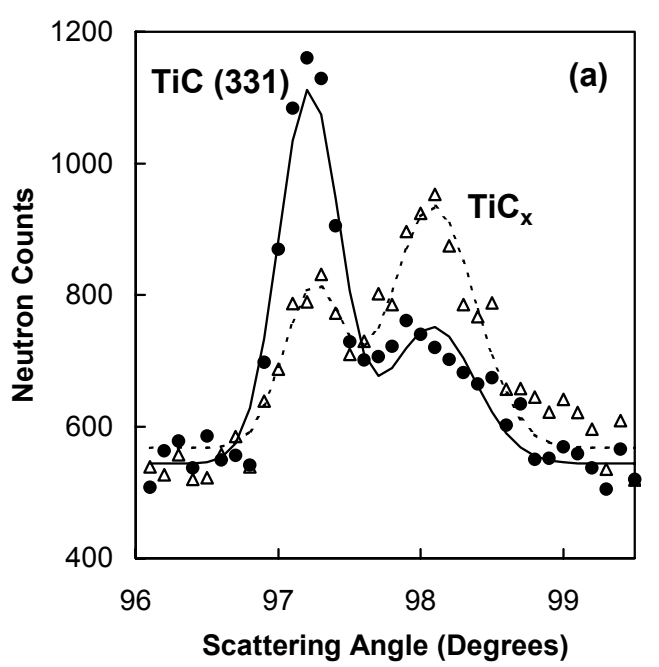

FIG. 1a. Distinct neutron diffraction peaks from two carbide phases, after $1 \mathrm{~h}(+)$ and after $16 \mathrm{~h}$ of sintering $(\Delta)$. Lines indicate fitted gaussian functions.

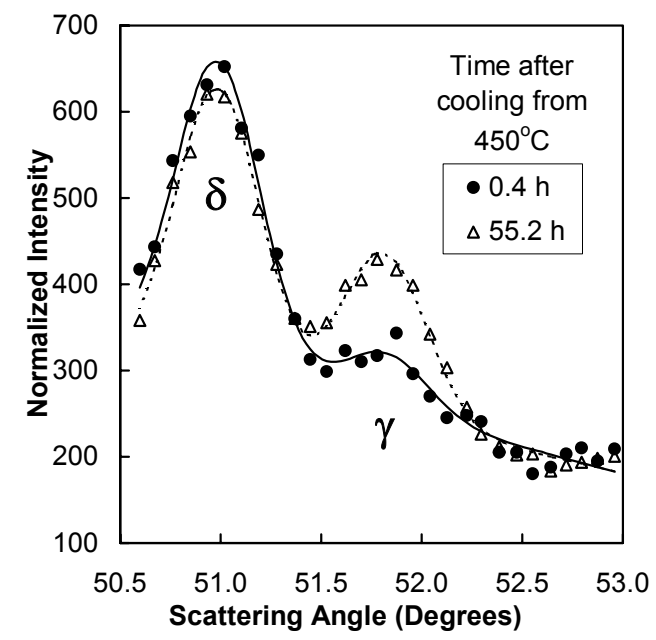

FIG. 2. Increasing quantity of $\gamma$-phase hydride over time, seen as growth of the (111) diffraction peak. The (111) $\delta$-phase peak decreases slightly. $\mathrm{T}=17^{\circ} \mathrm{C}$.

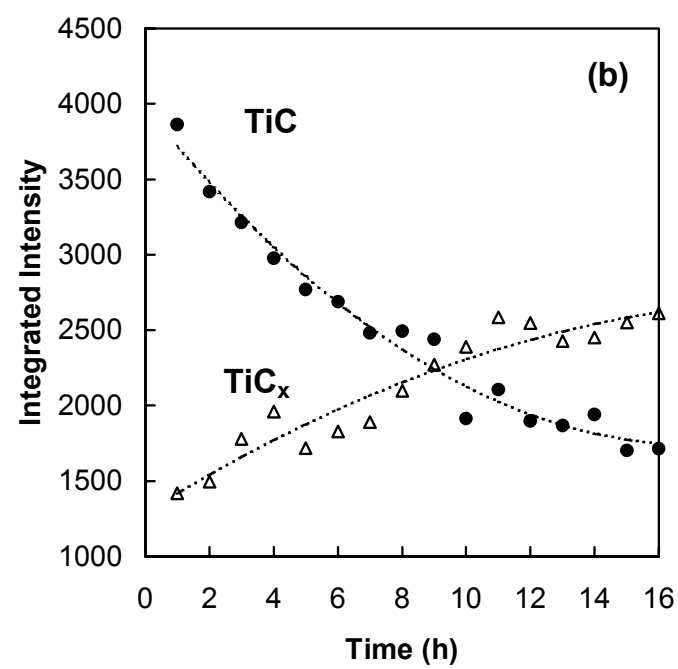

FIG. 1b. Erosion of $\mathrm{TiC}(+)$ with time and complementary growth of the carbondeficient phase $\operatorname{TiC}_{\mathrm{x}}(\Delta)$.

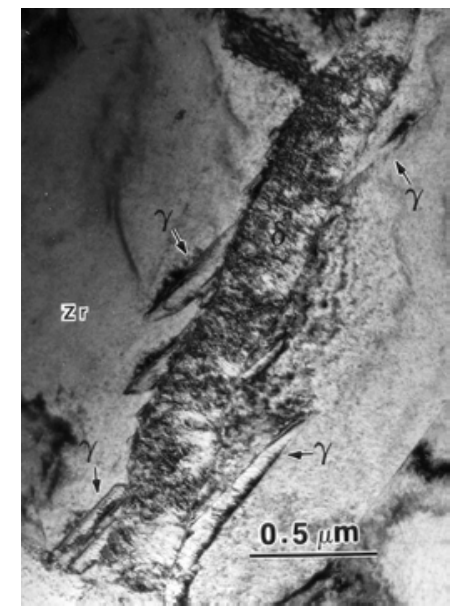

FIG. 3. TEM image of a $\delta$-phase hydride precipitate with $\gamma$-phase dendrites protruding from the outer boundary [9]. 